\title{
All-optical non-linear activation function for neuromorphic photonic computing using semiconductor Fano lasers
}

Rasmussen, Thorsten Svend; Yu, Yi; Mørk, Jesper

Published in:

Optics Letters

Link to article, DOI:

$10.1364 /$ OL.395235

Publication date:

2020

Document Version

Peer reviewed version

Link back to DTU Orbit

Citation (APA):

Rasmussen, T. S., Yu, Y., \& Mørk, J. (2020). All-optical non-linear activation function for neuromorphic photonic computing using semiconductor Fano lasers. Optics Letters, 45(14), 3844-3847.

https://doi.org/10.1364/OL.395235

\section{General rights}

Copyright and moral rights for the publications made accessible in the public portal are retained by the authors and/or other copyright owners and it is a condition of accessing publications that users recognise and abide by the legal requirements associated with these rights.

- Users may download and print one copy of any publication from the public portal for the purpose of private study or research.

- You may not further distribute the material or use it for any profit-making activity or commercial gain

- You may freely distribute the URL identifying the publication in the public portal

If you believe that this document breaches copyright please contact us providing details, and we will remove access to the work immediately and investigate your claim. 
To be published in Optics Letters:

Title: $\quad$ All-optical non-linear activation function for neuromorphic photonic computing using semiconductor Fano lasers

Authors: Thorsten Rasmussen, Yi Yu,Jesper Mork

Accepted: 05 June 20

Posted 08 June 20

DOI: $\quad$ https://doi.org/10.1364/OL.395235

(c) 2020 Optical Society of America 


\title{
All-optical non-linear activation function for neuromorphic photonic computing using semiconductor Fano lasers
}

\author{
Thorsten S. Rasmussen ${ }^{1}{ }^{1}$, , Y I YU ${ }^{1}$, AND Jesper Mork ${ }^{1}$ \\ ${ }^{1}$ DTU Fotonik, Technical University of Denmark, DK-2800 Kongens Lyngby, Denmark \\ *Corresponding author: thsv@fotonik.dtu.dk
}

Compiled June 2, 2020

We predict that semiconductor Fano lasers can be used to realize an all-optical non-linear activation function for neuromorphic photonic computing. By exploiting optical control of a Fano mirror, the laser can generate optical pulses with low threshold energy, gigahertz repetition rates and orders of magnitude suppression between the on- and off-states. Analytical estimates of the switching threshold energy, extinction ratio and refractory period agree well with numerical results. (๐) 2020 Optical Society of America

\section{http://dx.doi.org/10.1364/ao.XX.XXXXXX}

As digital electronics approaches a fundamental efficiency barrier, alternative, specialised computing strategies are increasingly being pursued [1]. These include neural networks for deep learning, with applications in e.g. real-time data handling, computer vision, and natural-language processing. Implementing neural networks using photonics-based architectures promises to increase computation speed by orders of magnitude, while simultaneously obtaining excellent energy efficiencies [1]. The artificial neurons making up the networks consist of two essential building blocks: a linear weighting and integration function (multiply and accumulate) to collect inputs from other neurons and a non-linear activation function (NLAF) to provide thresholding (figure 1(a)). The latter determines whether the neuron fires or not, through a sigmoid-like response with a suitable refractory period and output pulse generation [2]. These devices must simultaneously be compact, energy efficient, fast, and suitable for scalable integration, providing a challenging task [3]. Recently, there have been a number of suggestions for realising non-linear activation functions, including use of electro-absorption modulators [4] and micro-ring modulators [5], as well as a number of all-optical realisations [6-8]. An alternative option is to use excitable semiconductor lasers [1], for which Ref. [2] provides an extensive review.

In this work, we demonstrate how a specific type of microscopic laser, the so-called Fano laser [9], shows excitability and can be used to realise a nonlinear activation function for neuromorphic photonic computing, fulfilling the demands of thresholding (sigmoid-like response), possessing a nanosecond refractory period and generating optical pulses in response to input
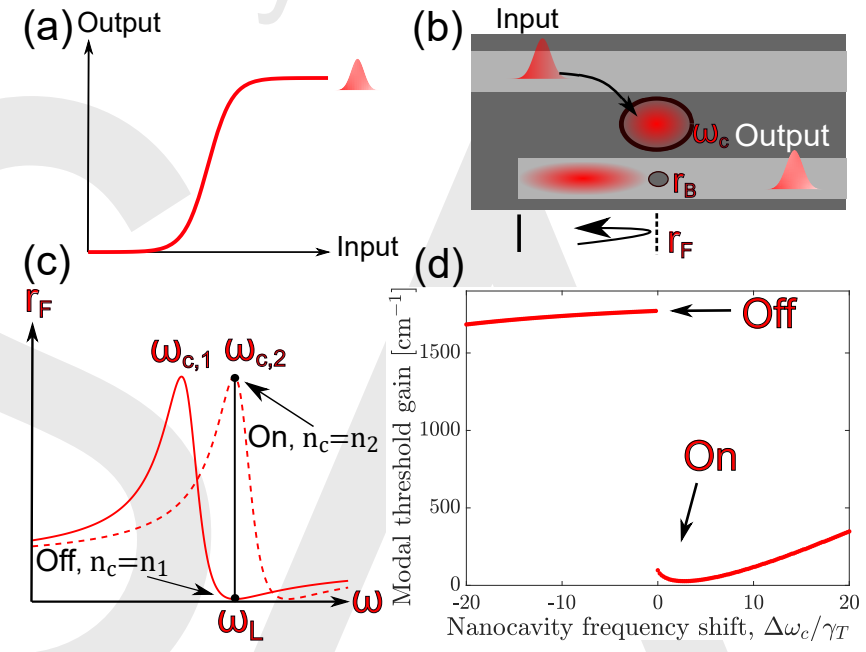

Fig. 1. (a) Schematic representation of a sigmoid-like nonlinear activation function. (b) Schematic of Fano laser operating as an NLAF with input and output ports. The right laser mirror, $r_{F}$, is formed by Fano resonance due to the nanocavity. (c) Fano reflection coefficient as function of frequency, demonstrating tuning between on and off-states by changing the nanocavity refractive index from $n_{1}$ to $n_{2}$. (d) Fano laser modal threshold gain as function of the nanocavity resonance frequency, showing the off and on-states corresponding to (c).

pulses with low energy consumption and an extremely small footprint, below $100 \mu \mathrm{m}^{2}$. The key characteristic of the Fano laser is that one or both of the laser mirrors are realised by Fano interference, through the interaction of a continuum of waveguide modes and a nearby discrete resonance [10], see figure 1(b). This leads to a narrowband reflectivity peaking near the resonance frequency of the defect nanocavity (figure 1(c)), with the characteristic asymmetric Fano shape, forming the laser mirror. By placing active material in the waveguide between the left mirror and the defect cavity, using buried heterostructure technology [11], a laser is formed, as indicated by the confined field (red) in figure 1(b). This device has previously been realised in a photonic crystal platform, showing single-mode lasing and self-pulsing [12,13], and predictions of a frequency modulation bandwidth beyond $1 \mathrm{THz}[14]$, as well as extraordinary 
resistance to external optical feedback [15]. Ref. 16 gives a comprehensive review of Fano laser research. The Fano mirror reflectivity is [16]

$$
r_{F}\left(\omega_{c}, \omega_{s}\right)=r_{B}+\left(i t_{B}-r_{B}\right) \frac{\gamma_{c}}{i\left(\omega_{c}-\omega_{L}\right)+\gamma_{T}}
$$

Here $r_{B}$ and $t_{B}$ are non-dispersive reflection and transmission coefficients of the partially transmitting element (PTE) in the waveguide (figure $1(\mathbf{b})), \gamma_{c}$ is the coupling rate from the waveguide to the nanocavity, $\gamma_{T}$ is the total decay rate of the nanocavity field, $\omega_{C}$ is the (tunable) resonance frequency of the nanocavity, and $\omega_{L}$ is the frequency of the incoming field. For $r_{B}>0$, the reflectivity is characterised by a small frequency separation between its extrema, which is ideal for applications in switching [17] and pulse generation. In particular, the narrowband reflectivity and highly dispersive phase give rise to a strong frequency dependence of the modal threshold gain $[9,14]$, see figure 1(d). States with large threshold gain separation (the onand off-states) are close in frequency, and can be switched between by tuning the nanocavity frequency. This enables the generation of short optical pulses using the scheme of active Q-switching [18]. If the resonance is initially in the off-state, the threshold gain is too high for the device to lase, meaning that a large population inversion can be built up through continuous pumping. The laser is then switched to the on-state, by tuning the resonance frequency of the nanocavity dynamically, at which point the population inversion strongly exceeds the threshold inversion. The excessive gain leads to a spike in the stimulated emission rate, and a strong pulse is emitted, depleting the carrier density. After pulse emission the nanocavity resonance relaxes back to the off-state, and the carrier density in the active region is restored.

Dynamical tuning of the refractive index in the nanocavity, and thus its resonance frequency, can be accomplished using e.g. the electro-optic effect, the Kerr effect, or the plasma effect due to excitation of free carriers. The first two require more complicated geometries, such as electrodes [19] or bow-ties for extreme field confinement[20], so in this work we study a Fano laser tuned by carrier dispersion and band filling in the nanocavity. The carriers are excited by two-photon absorption of an external optical pulse, as in e.g. [17, 21].

The laser dynamics are investigated numerically using our previously developed Fano laser time-domain model [16], but in an iterative and discretised version, as presented in Ref. 22 (Eqs. (2),(16), and (17) therein). The non-linearities in the nanocavity are implemented using Eq. (3) and (10) of Ref. 23 and the parameters therein, but with a single time constant $\tau_{c}$ for the carriers in the nanocavity. The external trigger pulse enters directly as an excitation in the equation for the nanocavity carrier density through the generation rate $G(t)$, representing either excitation via a pulse injected vertically from above the membrane, or sidecoupled excitation through an in-plane coupling waveguide on the other side of the nanocavity, i.e. the red input pulse in figure 1(a), with the only difference being in the excitation efficiency. Realistic parameter values based on our previously fabricated devices [12] are used, but with a buried heterostructure quantum well active region in the waveguide [11] instead of quantum dots throughout the sample. The parameters are, in the notation of [16]: $\alpha=3, \Gamma=0.12, v_{g}=c / n_{g}, n_{g}=3.17, g_{N}=1.3 \times 10^{-18}$ $\mathrm{m}^{2}, N_{0}=1 \times 10^{24} \mathrm{~m}^{-3}, r_{B}=0.4, \tau_{\text {in }}=270 \mathrm{fs}, \gamma_{c}=6 \times 10^{11}$ $\mathrm{s}^{-1}, \gamma_{T}=6.06 \times 10^{11} \mathrm{~s}^{-1}, R_{p}=3 R_{p, t h}, \tau_{\mathrm{s}}=\tau_{c}=0.28 \mathrm{~ns}$.

Figure 2(a) shows an example input excitation of the nanocavity and the corresponding laser output. It is clear that a threshold
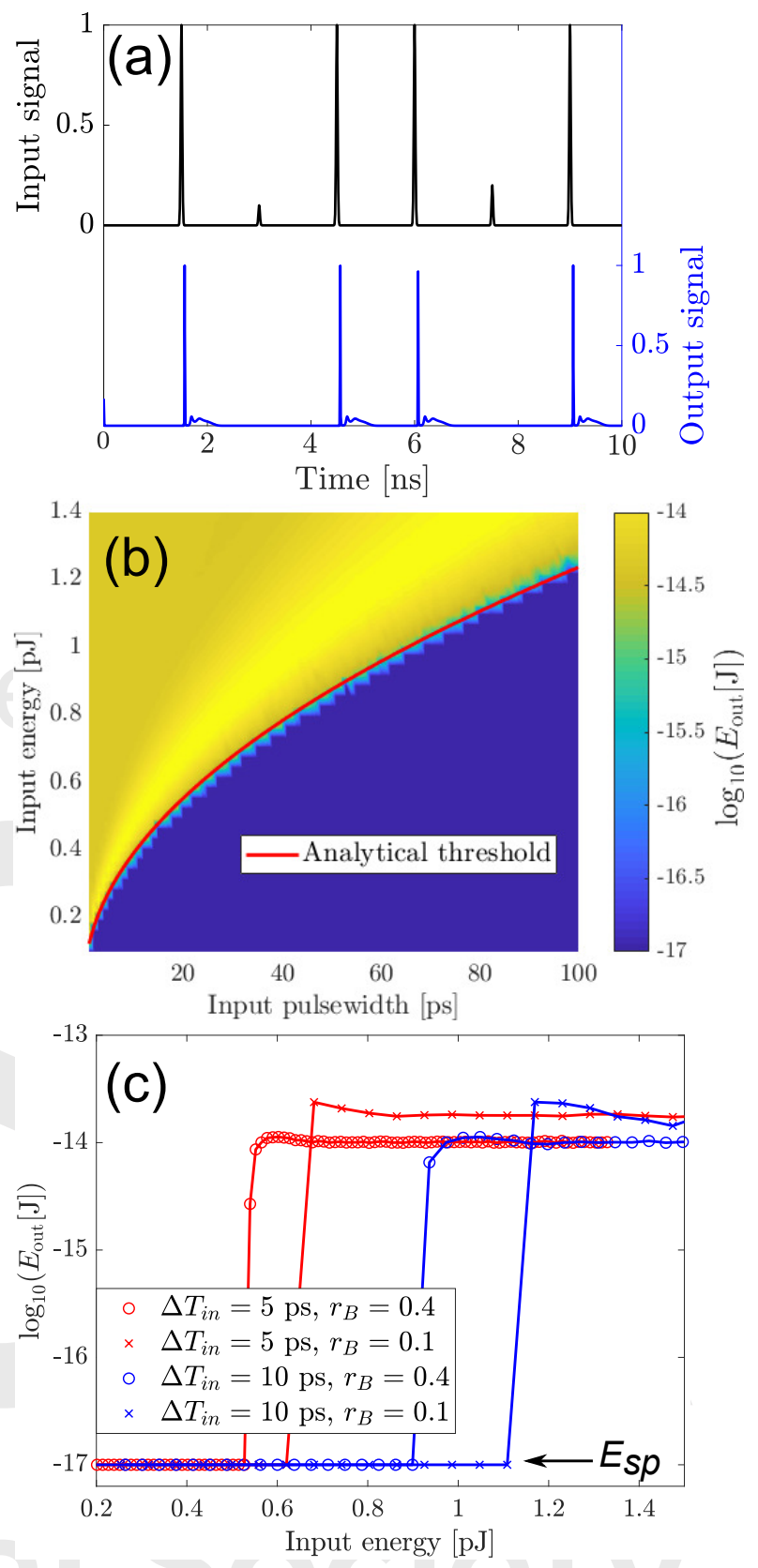

Fig. 2. (a) Calculated output dynamics for the given input signal, showing pulse generation and thresholding. (b) Output pulse energy (colour scale) as function of input pulsewidth and input pulse energy. The red curve is the prediction from Eq. (3). (c) Output pulse energy as function of input pulse energy for different input pulsewidths and PTE reflectivities. Solid lines are guides to the eye.

exists, beyond which well-defined optical pulses are generated. This is an example of the intrinsic excitability [2] of the Fano laser, and provides the thresholding mechanism of the non-linear activation function. Figure 2(b) presents a more systematic investigation, showing the output pulse energy (colour scale) as a function of the input pulse pulsewidth and pulse energy when operating at $R_{p}=3 R_{p, t h}$, where $R_{p, t h}$ is the laser threshold pump rate of the on-state at the minimum of the threshold gain in figure 1(d), with the pumping being either optical or electrical. For electrical pumping, the threshold current will be in the microamp range due to the small device size, leading to a low energy 
consumption. The red curve is an analytical estimate of the switching energy, given by Eq. (3), showing excellent agreement. This demonstrates clearly the desired sigmoid-like response, with only spontaneous emission noise below the threshold and output pulses in the $\approx 20 \mathrm{fJ}$ range beyond the threshold, resulting in an extinction ratio of several orders of magnitude.

This sigmoid-like response to tuning of the nanocavity resonance is intrinsic to the Fano laser Q-switching mechanism, because of the large difference in threshold gain and effective reflectivity between the off- and on-states. If the resonance tuning is insufficient to switch the laser from the off-state to the on-state, no light is emitted, as the laser cannot reach the required threshold gain. Instead, a sufficiently strong tuning of the resonance means that the laser reaches the on-state (crossing the gap in figure1(d)) and emits a pulse. The properties of this pulse are determined primarily by the off-state inversion, which means that once the threshold is crossed the pulse properties vary only weakly with the input, leading to a rapid plateau in the response. This dependence on the stationary threshold gain also gives the possibility to tune the response, in particular by adjustment of $\gamma_{T}, r_{B}$ and $\alpha$, which primarily define the sharpness of the threshold gain jump.

Figure 2(c) shows the output pulse energy as a function of the input pulse energy for different constant input pulsewidths. The threshold input energy decreases with a decreasing input pulsewidth, which is due to the two-photon absorption efficiency scaling with the square of the input peak power. All pulsewidths are seen to lead to similar input-output curves, with a small oscillation in the amplitude just beyond the threshold. The origin of this oscillation is variation in the effective reflectivity during pulse release, since it depends sensitively on the resonance frequency relative to the threshold gain minimum. As the input energy increases, the response levels out because the resonance overshoots the minimum consistently, and then releases the pulse during the decay of the carriers in the nanocavity.

The resonance tuning and input pulse energy required to release a pulse are, to first order, given by

$$
\begin{aligned}
\Delta \omega_{c, t h} & \simeq\left(\gamma_{T}-\frac{r_{B} \gamma_{T}^{2}}{\gamma_{c}}\right)\left(\frac{\gamma_{T} \tau_{i n}+1}{\gamma_{T} \tau_{i n}}\right) \\
E_{i n, t h} & \simeq \sqrt{\Delta T \frac{\Delta \omega_{c, t h} \gamma_{S}^{2}}{K_{C} G_{T P A}}}
\end{aligned}
$$

where $\tau_{\text {in }}$ is the laser roundtrip time, $K_{C}$ is the free carrier and band filling dispersion coefficient, $G_{T P A}$ is the two-photon generation coefficient, $\gamma_{S}$ is the inverse of the storage time of the excitation pulse in the nanocavity and $\Delta T$ is the input pulsewidth (FWHM). Eq. (2) is obtained by approximating the laser frequency and effective reflectivity as function of the detuning, and comparing with the off-state reflectivity $r_{B}$ to estimate the detuning distance between the on and off-states. It shows how the trigger energy can be lowered by reducing the contrast between the on- and off-states of the Fano mirror, i.e. increasing the PTEreflection $r_{B}$. It also shows that it is beneficial to increase the cavity length, as this reduces the longitudinal mode spacing of the laser, which in turn reduces the switching threshold power, with the additional benefit of reducing the laser threshold gain. Eq. (3) is obtained by converting the tuning threshold into an input pulse energy by assuming a Gaussian input pulse and that pulse release is much faster than the carrier lifetime.
The maximum obtainable extinction ratio is given by

$$
\Delta E_{\text {max }}=\frac{N\left(g_{\text {th }, o f f}\right) \hbar \omega_{L} V_{L C}}{E_{s p}}
$$

where $N$ is the off-state carrier density, as determined by the maximum off-state threshold gain $g_{\text {th,off }} \simeq \alpha_{i} / \Gamma+$ $1 /(2 L \Gamma) \ln \left(1 / r_{B}^{2}\right)$, which in turn is defined by the internal losses, $\alpha_{i}$, the field confinement factor, $\Gamma$, the cavity length, $L$, and $r_{B}$. $V_{L C}$ is the active region volume, and $E_{s p}$ is the integrated spontaneous emission power over the detection interval, giving the noise floor below the switching threshold in figures 2(b) and (c).

Eq. (2) and Eq. (4) expose an inherent trade-off for this device, exemplified in figure 2(c), where both output energy and threshold energy increase with a decrease in $r_{B}$. In general, changes that reduce the threshold switching energy tend to also reduce the extinction ratio, for instance increasing the PTE reflectivity $r_{B}$ or reducing the mode spacing through the cavity length or mirror linewidth. The explanation for this is simply that the obtainable off-state gain scales strongly with the spacing between modes, as achieving a low threshold gain for the Fano laser relies on aligning the nanocavity resonance with a longitudinal mode that fulfils the round-trip phase condition.

An intrinsic recovery time (refractory period) is present in the scheme due to two mechanisms. The first is that the tuning mechanism relies on the excitation of free carriers in the nanocavity, which decay with lifetime $\tau_{c}$. Secondly, the carrier density in the laser cavity is strongly depleted during pulse release, and has to be replenished afterwards, so that the slower of the two processes defines the actual recovery time. Figures 3(a)-(b) demonstrate this by comparing output dynamics for input excitations with different delay between trigger pulses. The secondary, closely spaced pulse $P_{2}$ results in only a small secondary output pulse, because the system has not yet reset. For the larger spacing shown in $P_{3}$, however, the first pulse is almost identically reproduced, because the input spacing is

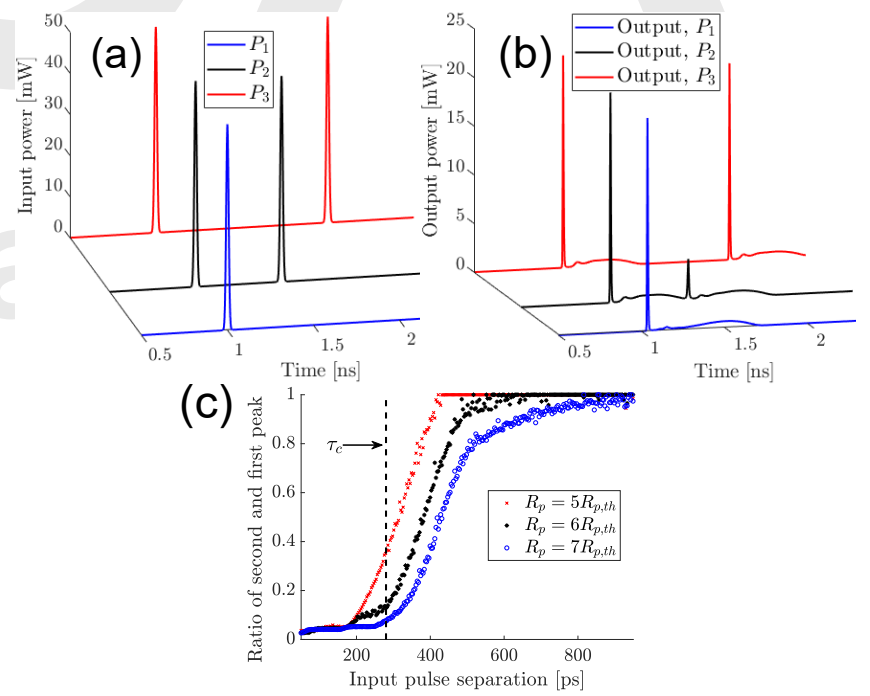

Fig. 3. (a) Three example excitation signals $P_{1}, P_{2}, P_{3}$. (b) Laser outputs corresponding to the input signals $P_{1}, P_{2}, P_{3}$. (c) Ratio of peak powers of the first and second pulse as function of input pulse separation, showing a clear refractory period, bounded from below by $\tau_{c}$ (vertical dashed line) and increasing with pump rate. 
approximately equal to the recovery time. This behaviour is quantified in more detail in figure 3(c), which shows the ratio of the secondary peak and the peak of the first output pulse in the time signal as a function of the time separation of two input pulses. Here, the ratio approaches zero for small separations, corresponding to essentially no secondary output pulse, until eventually converging to unity as the separation increases and the two pulses become independent. The refractory period is seen to increase with the pump rate, which may seem counterintuitive but is actually also the case in conventional Q-switching. The time to recover $90 \%$ of the initial carrier density, $N_{i}$, scales approximately as $t_{90} \simeq \tau_{s} \log \left[10\left(1-N_{f} / N_{i}\right)\right]$ where $\tau_{s}$ is the L-cavity carrier lifetime. The initial (off-state) carrier density, $N_{i}$, is directly proportional to the pump rate, while the final (after pulse release) carrier density, $N_{f}$, decreases with the pump rate, obeying approximately the conventional governing equations of active Q-switching [24]. As $N_{f}$ decreases and $N_{i}$ increases with pump rate, the recovery time consequently also increases, as seen in figure 3(c). This means that there is also a trade-off between maximising the extinction ratio and minimising the recovery time, and a suitable operation point must be chosen, depending on speed and energy requirements.

Regarding energy consumption and cascadability, the current scheme does not allow amplifier-free operation as the output pulse energy is roughly 1-2 orders of magnitude below the input energy. However, the inefficient energy conversion is due to the use of two-photon absorption for cavity tuning, and could be greatly improved by using linear absorption instead. Thus, improvements in nanofabrication technology, enabling growth of a localised buried heterostructure of larger band gap within the nanocavity, and thus excitation by linear absorption, will dramatically improve the efficiency and reduce energy consumption down to trigger energies of tens of femtojoules. In that case, amplifier-free cascadability in an all optical on-chip network based on Fano laser structures would be feasible, providing that a suitable multiply-accumulate system could be designed within the platform, leading to extreme miniaturisation and ultra-low energy consumption.

It is also of interest to explore applications of this scheme simply as an ultra-compact, low-energy and high-speed non-linear activation function to be incorporated into existing on-chip realisations. This still requires integration of the lasers on silicon and efficient coupling from the multiply-accumulate elements to the Fano laser non-linear activation function, likely to result in a trade-off between energy consumption and device footprint. In this context, we note the promising progress of integration of photonic crystal lasers on silicon [25, 26]. Finally, further research into hybrid electro-optical schemes with electrical control of the nanocavity resonance is also warranted, since this would allow incorporation of multiply-accumulate functionality simply by introduction of integrated photodetectors. Given the notable electro-optic effect in indium phosphide, this would likely also enable amplifier-free cascadability, greatly enhancing the potential applicability.

In conclusion, it has been shown that an all-optical non-linear activation function for neuromorphic photonics can be realised using photonic crystal Fano lasers. The Fano laser system is inherently excitable and has a refractory period enabling $\mathrm{GHz}$ operation with several orders of magnitude suppression between the on- and off-states. Simultaneously, the photonic crystal platform allows for strong miniaturisation of the components, yielding ultra-small footprints in the range of $100 \mu \mathrm{m}^{2}$ and small switching energies in the $100 \mathrm{fJ}$ range. Fano lasers are thus promising candidates for ultra-fast, compact, on-chip implementations of neuromorphic photonic computing schemes.

Funding Information. Villum Fonden (8692); European Research Council (Grant No. 834410 Fano); Danish National Research Foundation (Grant No. DNRF147).

Disclosures. The authors declare no conflicts of interest.

\section{REFERENCES}

1. B. J. Shastri, A. N. Tait, T. Ferreira de Lima, M. A. Nahmias, H.-T. Peng, and P. R. Prucnal, Encycl. Complex. Syst. Sci. p. 1-37 (2018).

2. P. R. Prucnal, B. J. Shastri, T. F. de Lima, M. A. Nahmias, and A. N. Tait, Adv. Opt. Photonics 8, 228 (2016).

3. M. Miscuglio, G. C. Adam, D. Kuzum, and V. J. Sorger, APL Mater. 7, 100903 (2019).

4. R. Amin, J. George, S. Sun, T. Ferreira de Lima, A. N. Tait, J. Khurgin, M. Miscuglio, B. J. Shastri, P. R. Prucnal, T. El-Ghazawi et al., APL Mater. 7, 081112 (2019).

5. A. N. Tait, T. F. De Lima, M. A. Nahmias, H. B. Miller, H.-T. Peng, B. J. Shastri, and P. R. Prucnal, Phys. Rev. Appl. 11, 064043 (2019).

6. M. Miscuglio, A. Mehrabian, Z. Hu, S. I. Azzam, J. George, A. V. Kildishev, M. Pelton, and V. J. Sorger, Opt. Mater. Express 8, 3851 (2018).

7. G. Mourgias-Alexandris, A. Tsakyridis, N. Passalis, A. Tefas, K. Vyrsokinos, and N. Pleros, Opt. express 27, 9620 (2019).

8. Y. Zuo, B. Li, Y. Zhao, Y. Jiang, Y.-C. Chen, P. Chen, G.-B. Jo, J. Liu, and S. Du, Optica 6, 1132 (2019).

9. J. Mork, Y. Chen, and M. Heuck, Phys. Rev. Lett. 113, 163901 (2014).

10. A. E. Miroshnichenko, S. Flach, and Y. S. Kivshar, Rev. Mod. Phys. 82, 2257 (2010).

11. A. Sakanas, Y. Yu, E. Semenova, L. Ottaviano, H. K. Sahoo, J. Mork, and K. Yvind, "Fabrication and experimental demonstration of photonic crystal laser with buried heterostructure," in 2017 CLEO/Europe-EQEC, (IEEE, 2017).

12. Y. Yu, W. Xue, E. Semenova, K. Yvind, and J. Mork, Nat Photon 11, 81 (2017). Letter.

13. T. S. Rasmussen, Y. Yu, and J. Mork, Laser \& Photonics Rev. 11, 1700089 (2017).

14. T. S. Rasmussen, Y. Yu, and J. Mork, Opt. Express 26, 16365 (2018).

15. T. S. Rasmussen, Y. Yu, and J. Mork, Phys. Rev. Lett. 123, 233904 (2019).

16. J. Mork, Y. Yu, T. S. Rasmussen, E. Semenova, and K. Yvind, IEEE J. Sel. Top. Quantum Electron. 25, 1 (2019).

17. D. Bekele, Y. Yu, K. Yvind, and J. Mork, Laser \& Photonics Rev. 13, 1900054 (2019).

18. W. G. Wagner and B. A. Lengyel, J. Appl. Phys. 34, 2040 (1963).

19. L.-D. Haret, X. Checoury, F. Bayle, N. Cazier, P. Boucaud, S. Combrié, and A. de Rossi, Opt. Express 21, 10324 (2013).

20. S. Hu, M. Khater, R. Salas-Montiel, E. Kratschmer, S. Engelmann, W. M. Green, and S. M. Weiss, Sci. advances 4 (2018).

21. T. Tanabe, M. Notomi, H. Taniyama, and E. Kuramochi, Phys. Rev. Lett. 102, 043907 (2009)

22. T. S. Rasmussen, Y. Yu, and J. Mork, "Photonic crystal laser based on fano interference allows for ultrafast frequency modulation in the thz range," in Proc. SPIE, , vol. 10939 (2019).

23. Y. Yu, E. Palushani, M. Heuck, N. Kuznetsova, P. T. Kristensen, S. Ek, D. Vukovic, C. Peucheret, L. K. Oxenløwe, S. Combrié, A. de Rossi, K. Yvind, and J. Mørk, Opt. Express 21, 31047 (2013).

24. A. E. Siegman, Lasers (University Science Books, 1986).

25. G. Crosnier, D. Sanchez, S. Bouchoule, P. Monnier, G. Beaudoin, I. Sagnes, R. Raj, and F. Raineri, Nat. Photonics 11 (2017).

26. M. Takiguchi, A. Yokoo, K. Nozaki, M. D. Birowosuto, K. Tateno, G. Zhang, E. Kuramochi, A. Shinya, and M. Notomi, APL photonics 2, 046106 (2017). 


\section{FULL REFERENCES}

1. B. J. Shastri, A. N. Tait, T. Ferreira de Lima, M. A. Nahmias, H.-T. Peng, and P. R. Prucnal, "Neuromorphic photonics, principles of," Encycl. Complex. Syst. Sci. p. 1-37 (2018).

2. P. R. Prucnal, B. J. Shastri, T. F. de Lima, M. A. Nahmias, and A. N. Tait, "Recent progress in semiconductor excitable lasers for photonic spike processing," Adv. Opt. Photonics 8, 228-299 (2016).

3. M. Miscuglio, G. C. Adam, D. Kuzum, and V. J. Sorger, "Roadmap on material-function mapping for photonic-electronic hybrid neural networks," APL Mater. 7, 100903 (2019).

4. R. Amin, J. George, S. Sun, T. Ferreira de Lima, A. N. Tait, J. Khurgin, M. Miscuglio, B. J. Shastri, P. R. Prucnal, T. El-Ghazawi et al., "Ito-based electro-absorption modulator for photonic neural activation function," APL Mater. 7, 081112 (2019).

5. A. N. Tait, T. F. De Lima, M. A. Nahmias, H. B. Miller, H.-T. Peng, B. J. Shastri, and P. R. Prucnal, "Silicon photonic modulator neuron," Phys. Rev. Appl. 11, 064043 (2019).

6. M. Miscuglio, A. Mehrabian, Z. Hu, S. I. Azzam, J. George, A. V. Kildishev, M. Pelton, and V. J. Sorger, "All-optical nonlinear activation function for photonic neural networks," Opt. Mater. Express 8, 38513863 (2018).

7. G. Mourgias-Alexandris, A. Tsakyridis, N. Passalis, A. Tefas, K. Vyrsokinos, and N. Pleros, "An all-optical neuron with sigmoid activation function," Opt. express 27, 9620-9630 (2019).

8. Y. Zuo, B. Li, Y. Zhao, Y. Jiang, Y.-C. Chen, P. Chen, G.-B. Jo, J. Liu, and S. Du, "All-optical neural network with nonlinear activation functions," Optica 6, 1132-1137 (2019).

9. J. Mork, Y. Chen, and M. Heuck, "Photonic crystal fano laser: Terahertz modulation and ultrashort pulse generation," Phys. Rev. Lett. 113, 163901 (2014).

10. A. E. Miroshnichenko, S. Flach, and Y. S. Kivshar, "Fano resonances in nanoscale structures," Rev. Mod. Phys. 82, 2257-2298 (2010).

11. A. Sakanas, Y. Yu, E. Semenova, L. Ottaviano, H. K. Sahoo, J. Mork, and K. Yvind, "Fabrication and experimental demonstration of photonic crystal laser with buried heterostructure," in 2017 CLEO/Europe-EQEC, (IEEE, 2017).

12. Y. Yu, W. Xue, E. Semenova, K. Yvind, and J. Mork, "Demonstration of a self-pulsing photonic crystal fano laser," Nat Photon 11, 81-84 (2017). Letter.

13. T. S. Rasmussen, Y. Yu, and J. Mork, "Theory of self-pulsing in photonic crystal fano lasers," Laser \& Photonics Rev. 11, 1700089 (2017).

14. T. S. Rasmussen, Y. Yu, and J. Mork, "Modes, stability, and smallsignal response of photonic crystal fano lasers," Opt. Express 26, 16365-16376 (2018).

15. T. S. Rasmussen, Y. Yu, and J. Mork, "Suppression of coherence collapse in semiconductor fano lasers," Phys. Rev. Lett. 123, 233904 (2019).

16. J. Mork, Y. Yu, T. S. Rasmussen, E. Semenova, and K. Yvind, "Semiconductor fano lasers," IEEE J. Sel. Top. Quantum Electron. 25, 1-14 (2019).

17. D. Bekele, Y. Yu, K. Yvind, and J. Mork, "In-plane photonic crystal devices using fano resonances," Laser \& Photonics Rev. 13, 1900054 (2019).

18. W. G. Wagner and B. A. Lengyel, "Evolution of the giant pulse in a laser," J. Appl. Phys. 34, 2040-2046 (1963).

19. L.-D. Haret, X. Checoury, F. Bayle, N. Cazier, P. Boucaud, S. Combrié, and A. de Rossi, "Schottky msm junctions for carrier depletion in silicon photonic crystal microcavities," Opt. Express 21, 10324-10334 (2013).

20. S. Hu, M. Khater, R. Salas-Montiel, E. Kratschmer, S. Engelmann, W. M. Green, and S. M. Weiss, "Experimental realization of deepsubwavelength confinement in dielectric optical resonators," Sci. advances 4 (2018).

21. T. Tanabe, M. Notomi, H. Taniyama, and E. Kuramochi, "Dynamic release of trapped light from an ultrahigh- $q$ nanocavity via adiabatic frequency tuning," Phys. Rev. Lett. 102, 043907 (2009).

22. A. E. Siegman, Lasers (University Science Books, 1986).

23. G. Crosnier, D. Sanchez, S. Bouchoule, P. Monnier, G. Beaudoin, I. Sagnes, R. Raj, and F. Raineri, "Hybrid indium phosphide-on-silicon nanolaser diode," Nat. Photonics 11 (2017).

24. M. Takiguchi, A. Yokoo, K. Nozaki, M. D. Birowosuto, K. Tateno, G. Zhang, E. Kuramochi, A. Shinya, and M. Notomi, "Continuous-wave operation and 10-gb/s direct modulation of inasp/inp sub-wavelength nanowire laser on silicon photonic crystal," APL photonics 2, 046106 (2017).
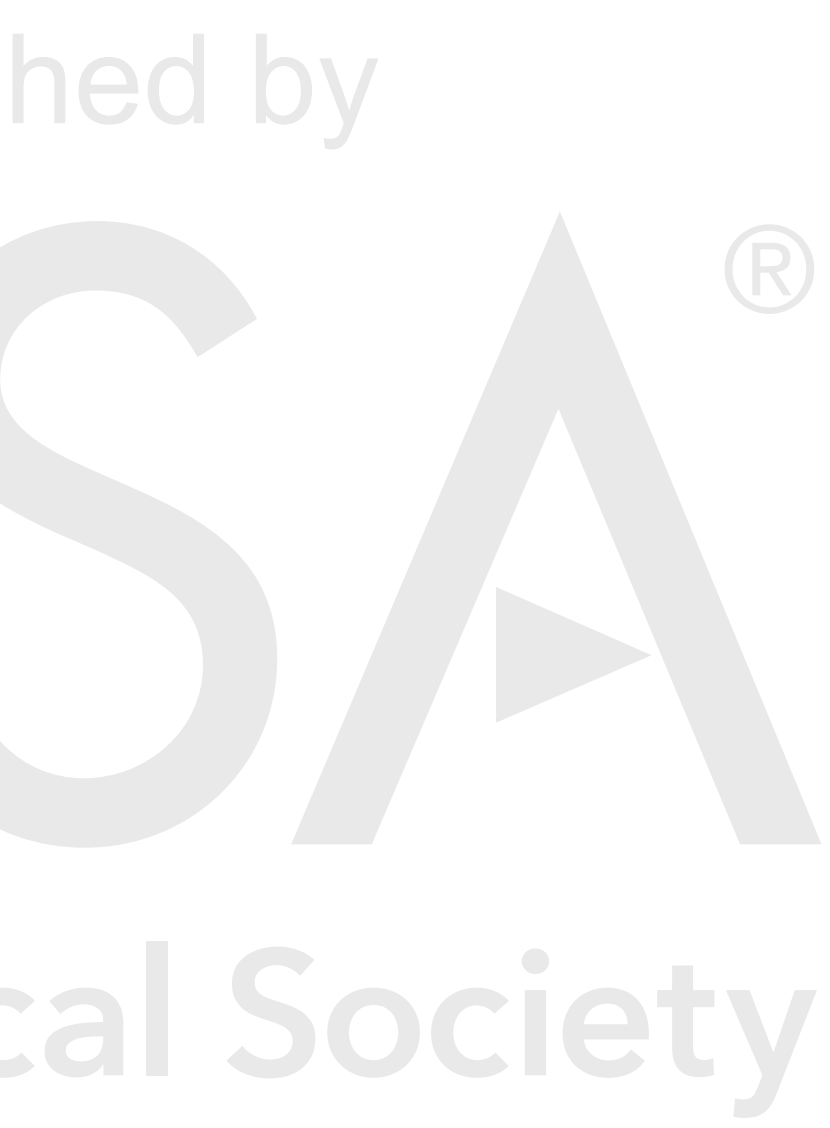